\title{
A DEVELOPED NETWORK LAYER HANDOVER BASED WIRELESS NETWORKS
}

\section{Ali Safa Sadiq ${ }^{1}$, Kamalrulnizam Abu Bakar ${ }^{2}$, Kayhan Zrar Ghafoor ${ }^{3}$, Jaime Lloret $^{4}$ and Mohammed Adam Ibrahim Fakhreldin ${ }^{1}$}

\author{
${ }^{1}$ Faculty of Computer systems \& Software Engineering, Universiti Malaysia Pahang, \\ 26300 Gambang, Pahang, Malaysia \\ ${ }^{2}$ Faculty of Computing, Universiti Teknologi Malaysia,81310 UTM Skudai, \\ Johor D. T, Malaysia \\ ${ }^{3}$ Faculty of Engineering, University of Koya, Daniel Miterrand Boulevard, Koya, KOY45, \\ Kurdistan Region-IRAQ \\ ${ }^{4}$ Departamento de Comunicaciones, Universidad Politecnica, de Valencia Camino de Vera s/n, \\ 46022, Valencia, Spain \\ Email: alisafasadiq@ump.edu.my
}

\begin{abstract}
This paper proposes an Advanced Mobility Handover (AMH) scheme based on Wireless Local Area Networks (WLANs) by developing a network layer handover procedure which triggers messages to be sent to the next access point. The proposed AMH scheme performs the network handover process, which is represented by binding update procedure in advance during the time mobile node is still connected to the current AP in the link layer. Furthermore, a unique home IPv6 address is developed to maintain an IP communication with other corresponding nodes without a care-of-address during mobile node $\$$ ' $\$$ s roaming process. This can contribute significantly to reducing network layer handover delays and signaling costs by eliminate the process of obtaining a new care-of-address and processing the handover of network layer in advance while the mobile node is still communicating with the current access point. Eventually, the conducted OMNET++ simulated scenario shows that the proposed AMH scheme performs the best in terms of reducing the handover delay as compared to the state of the art.
\end{abstract}

Keywords: Advanced Mobility Handover; Unique IPv6 Address; Low Handover Delay; RealTime.

\section{INTRODUCTION}

Nowadays people are more concerning on wireless Internet access for entertainment purposes. For example, a Voice-over-IP (VoIP) calls, radio and Television-over-IP (ToIP) services while they are travailing on cars, buses, high speed trains or airplanes. This fast development of wireless Internet leads to a request for high speed network access. Thus, different mobility management protocols are introduced as a way to provide IP connectivity any time and any place with high Quality-of-Service (QoS).

In wireless networks when Mobile Node (MN) moves from one place to another, a user can be connected through the wireless access link but, cannot move without the loss of the IP communication. As a result, in order to keep the routability between $\mathrm{MN}$ and Access Routers (AR), the IETF has designed Mobile Internet Protocol MIP to provide mobility in mobile devices. This mobility extension was developed based on IEEE 802.11 Brenner (1992) standard 
is 802.11 ac which can provide more than 1000 Mbps. Thus, by employing MIP MN could achieve a mobility management system.

MIP version 6 (MIPv6) is designed by IETF as a way to maintain network layer handover and to redirect the traffic between two Access Points (AP) in the wireless network, known as a Foreign Agent (FA) and Home Agent (HA). When an MN has moved out of its current network, the duties of FA is informing the HA the new Care-of-Address (CoA) of MN. In contrast, the HA is performing as a globally-static route to the Correspondent Nodes (CN) for MN. The MN expected to be supplied with IPv6 address whether it is currently connected to HA' ARs or other networks with different ARs configured with different global routing prefixes. The home address is an IPv6 address that assigned by HA of MN which is started with the home subnet prefix of HA. During the time MN is connected to its HA, all the traffic of packets are addressed to its configured home address. Afterwards, all the packets that were sent by CNs are routed to the MN's HA employing the typical Internet routing mechanisms Johnson (2004).

On the other hand, when an $\mathrm{MN}$ is connected to some foreign networks, it utilizes one or more of CoAs; that is depending on the number of visited foreign networks. The assigned CoA is an IPv6 address with a subnet prefix indicating the visited foreign network. By the time MN is active with a particular foreign network, the packets are addressed with the obtained CoA to be reachable to the MN. Moreover, in some cases, MN can accept packets from a number of CoAs, for instance when it is roaming but still accessible at the previous network.

The main concept of MN's addressability that achieved by MIPv6 is the binding process. As long as the $\mathrm{MN}$ is away from its HA, the home address that is already registered in the AR of $\mathrm{HA}$, in addition to one or more of CoAs are used to send and receive packets between $\mathrm{MN}$ and $\mathrm{CN}$. When the HA received the Binding Update (BU) message that was sent by MN, it replies by sending a Binding Acknowledgment message. Eventually, when the acknowledgment message is received within specified lifetime, the $\mathrm{MN}$ uses the registered CoA as the source of the packet, and there will be no need to use the Home Address option in the packet.

Although, in MIPv6, there are some issues still need to be addressed regarding to mobility handover process. When MN performs a fast movement, the signal quality of the current AP can go down rapidly. Thus, $\mathrm{MN}$ is not able to connect for a long period with the Current AR (CAR). For instance, when MN does not have sufficient time to send handover signals which are responsible for new IP address registration process, MN will face high mobility handover delay. Thus, it can lead to mobility handover failure. In other words, the mobility handover process which are performed by MN to obtain a new CoA from visited network, in addition to the registration processes with HA are negatively affected by inefficient mobility handover process. Consequently it can degrade the QoS of ongoing applications due to high overall handover delay. In this regards, several approaches have been proposed as a way to reduce the handover latency that experienced within MIPv6 due to IPv6 address configuration and registration processes.

\section{RELATED WORKS}

One of the essential issues in wireless communications is the handover delay. Hence, many studies have tried to overcome with appropriate solution to decrease the associated time delay during handover process. In order to maintain the mobility handover process of network layer, firstly the Internet Engineering Task Force (IETF) has proposed various IP-based mobility management schemes, such as MIPv6, Hierarchical Mobile IPv6 (HMIPv6), and Fast MIPv6 (FMIPv6) (Perkins2002; Johnson2011; Soliman2008; Jang2008). The goal of these schemes is to maintain the communication continuity when a MN moves between different ARs (Lee2010; 
Lee2013). However, these mobility protocols still have shortcomings in terms of handover latency, packet loss, signaling overheads Lee (2013).

In MIPv6 the handover detection can be considered as an essential issue which leads MN does not have the ability to process the potential mobility handover in before the current connection is disconnected. This can help in decreasing the overall latency that related to handover processes by performing the mobility handover in advance way before the Received Signal Strength (RSS) of current AP goes below the threshold. This can be achieved through maintain the new CoA obtaining and BU registration processes during the link layer handover procedure. As a consequence, MN with MIPv6 faces problems due to inefficient mobility handover processes. That is, when the network connection with current access link is lost and all related network layer signaling processes need to be postponed after the link layer handover is achieved with new AP. Thus, the overall handover time delays in addition to signaling costs are increased consequently during the handovers (Macha'n2010; Senan2011; Cho2013).

In contrast, a network based mobility management protocol Proxy MIPv6 (PMIPv6) (Gundavelli, 2008) is developed as a way to reduce the signaling overhead of MIPv6. Two new elements are introduced by PMIPv6, Mobility Access Gateway (MAG) and Local Mobility Anchor (LMA). The updating process of MN\$\$s movement is identified by the MAG, that is a function located at the AR. However, PMIPv6 was developed mainly for the local mobility management, which does not support a global mobility management (Lee2010; Giaretta2012). For instance, when some MNs support only MIPv6 and intending to use PMIPv6, the network infrastructure should provide MIPv6 functions to them. Therefore, the MAG in this case cannot act like the HA. The MAG suppose to advertise its own routing prefix instead of the IPv6 prefix that the MN always receives when connect to any AP within PMIPv6 domain (MN-HoA).

Although, some approaches recently are tried to enhance the functionality of PMIPv6, until now cannot support the seamless mobility handover (Lee 2010; Lee 2013). The reason is that, within PMIPv6 a high handover delay is normally experienced by inter-subnet handover. Thus, high packet loss could be occurred especially with real-time applications due to the high packet rate. Moreover, in case of any single failure of a MAG can degrade the performance to be worst than standard MIPv6.

An Improved Fast Handover scheme for Hierarchical Mobile IPv6 (IF-HMIPv6) was proposed in (Chen-wen, 2009) in order to reduce the handover delay and packet loss. However, the time that was consumed in address configuration process of CoA inside one local MAP still visible. Moreover, this study did not discuss the consequences behind increasing the number of MNs. In other words, the negative effects which lead to signaling overhead then decrease the QoS ongoing applications due to high number of MNs.

On the other hand, a Seamless Flow Mobility Management Architecture (SFMMA) for vehicular communication networks was proposed in Meneguette (2013). The proposed SFMMA method was introduced as a way to cope with the mobility management aspects in Vehicular Ad Hoc Networks (VANETs), in order to maintain the wireless connection with roadside network service nodes during vehicular movement. However, at best, by using the SFMMA scheme MN still asking information from the MAG where the network interface is connected; inquires about network status and the home network prefixes (HNP). Throughout this information, MN can indicate whether the interface is still active or not, otherwise it does not know to whom it needs to route the packets, and what are the prefixes that can be used to send its packets. 


\section{DEVELOPED NETWORK LAYER HANDOVER ALGORITHM}

In this section the detailed design of the AMH scheme is discussed by illustrating perspective architecture of AMH to explain how the handover process is performed. After the handover decision with one particular AP is taken, the AMH scheme will be triggered by MN. The triggering message is enclosed in an association request frame during the IEEE 802.11/b handover process. The AMH process is triggered as a way to inform the HA and $\mathrm{CN}$ the changes in the network layer (AR) of the wireless networks through the new selected AP. In AMH architecture there are ARs connected to the Internet Service Provider (ISP) which relies on IPv6, APs, and MN being mobile across the deployed APs which is illustrated in Figure 1. These APs can access the Internet connection through ARs. Figure 1 shows the mobility scenario when MN crosses different APs and different ARs. During the roaming process the MN uses a unique home IPv6 address assigned by HA in order to keep traffic flowing without a CoA. Therefore, the proposed AMH scheme introduces a novel unique IPv6 address which can be used by the MN during the roaming process.

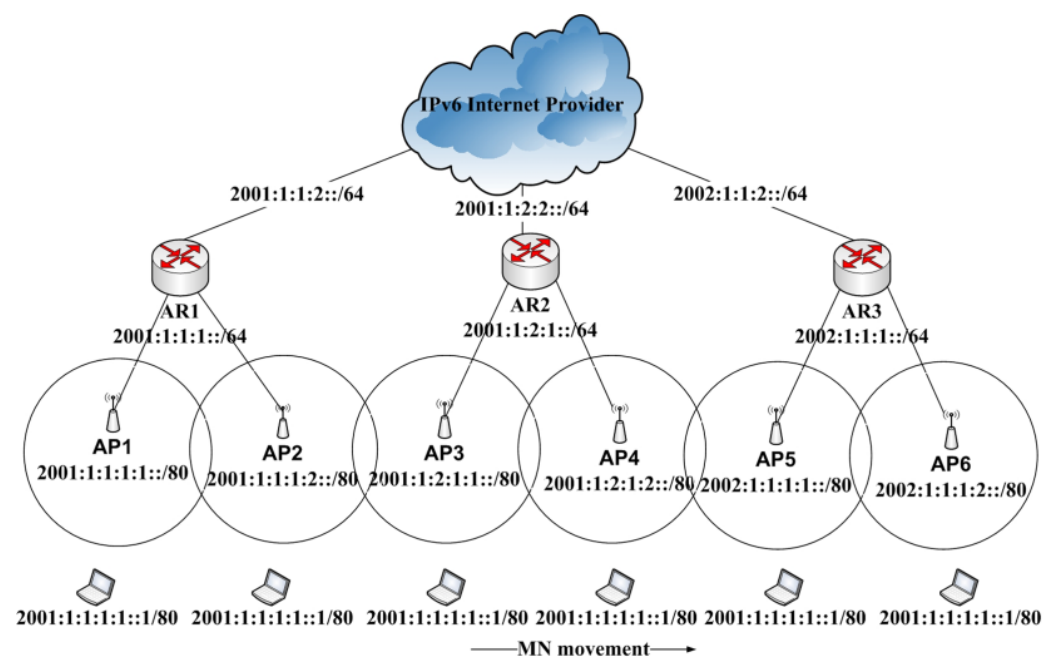

Figure 1. Proposed MIPv6 Addressing Scenario For AMH

These unique IPv6 addresses can be achieved by including three global routing prefix fields, AP ID, and MN ID in the IPv6 address of any MN and AP. The IPv6 addresses have been configured in each AP by first assigning the global routing prefix, which is part of the IPv6 address in all APs which are connected to the same AR. The second part of the AP's IPv6 address is the AP ID which uniquely identifies each AP and the third part of the IPv6 address is the MN ID in AP, which is assigned to zero. On the other hand, the IPv6 address for each MN is uniquely identified by HA using the MN ID field, which is set to 16 bits in the new IPv6 format. Therefore, when crossing different subnets during the roaming process, the MN does not need to use CoA from the visited network while employing the proposed unique IPv6 address from its HA as shown in Figure 1. On the contrary, when MN visits a new network with a different a global routing prefix, the MN keeps communicating with the new AP and AR with the same HA's unique IPv6 address using the MN ID. This mobility handover case can occur when the MN performs the handover with an AP which belongs to either (AR1 or AR2) and AP associated to (AR3) with different global routing prefixes as shown in Figure 1.

Figure 2 shows the IPv6 address format and data structure in table form saved in each HA, AR, and AP in the proposed AMH scheme. Three IPv6 fields (IPv6 address format) are illustrated in Figure 2 where $i$ is set to 16 bits. Moreover, the data structure in table form is 
shown in Figure 2. This table is designed and integrated into the proposed AMH in order to ensure that the IPv6 address of AP and AR during the MN roaming process is updated. These tables are utilized in AP, AR, and HA. Figure 2 shows the data structure in table form, known as (local MN table), which has been saved in HA. This local MN table in HA contains the MN's IPv6 address along with the IPv6 address of the AR that the MN is currently connected with as shown in Figure 2. The routing table in each AR (MN routing table) consists of the IPv6 address of the MN with MN's ID in case the MN is attached to an AR with a different global routing prefix, and AP's IPv6 address which the MN is currently associated with as shown in Figure 2. Finally, in each particular AP the table of the neighbor of AP's IPv6 and MAC address, current associated MNs IPv6 addresses, and MN's ID for each MN created by AP after the association process are saved as a table as shown in Figure 2.

In order for the MN to trigger the AMH scheme in the time the handover decision has been taken, the AMH message has been developed. In standard 802.11/b there are various frame types defined to be used by MN and AP for communications, as well as managing and controlling the wireless link. The association request frame in Standard 802.11/b is sent by MN to $\mathrm{AP}$ in order to allocate resources for the $\mathrm{MN}$ and to synchronize with a radio Network Interface Card (NIC). The association request frame is a management frame as defined in Society, (2007). Basically, the frame body of a management frame of subtype Association Request contains information about the MN such as: supported data rates, the SSID of the network the MN intends to associate with, and some other information. In the proposed AMH scheme the association request frame's body is modified by adding 130 bits. The IPv6 address of the MN uses 128 bits in each association frame plus 2 bits for the AMH triggering the AMH message.

In the proposed $\mathrm{AMH}$ architecture three handover cases are considered which are: handovers within the same subnet (AMH1), with different subnets and same global routing prefix (AMH2) and with different subnets and different global routing prefix (AMH3). The IPv6 address of the $\mathrm{MN}$ is included in the body of association request frame each time a request is sent to the new selected AP. Moreover, the AMH message code included in each association request frame is sent by the $\mathrm{MN}$ in order to trigger the $\mathrm{AMH}$ scheme by the selected AP. Table 1 shows the three types of association request frame sent by the $\mathrm{MN}$ and the $\mathrm{AMH}$ procedure which is executed by the next AP. When MN includes the AMH code (01) a request is made of the selected AP to reserve memory space, establish the MN ID which will later be used as a packet's address which is directed to the MN. Thus, this created MN ID will be used to send and receive traffic between the MN and AP. 




Figure 2. The format of the proposed unique IPv6 address and data structure as a table form saving in each of HA, AR, and AP in the proposed AMH scheme.

Table 1. The Contents of Modified Association Request Frame Send by MN and AMH Procedure Process by Next AP

\begin{tabular}{|l|l|}
\hline Body Information & AMH \\
\hline Default information + MN IPv6 address + (01) & reserve memory space, establish MN ID, and execute AMH1 \\
\hline Default information + MN IPv6 address + (10) & reserve memory space, establish MN ID, and execute AMH2 \\
\hline Default information + MN IPv6 address + (11) & reserve memory space, establish MN ID, and execute AMH3 \\
\hline
\end{tabular}

\section{PERFORMANCE EVALUATION}

Table 2 provides summarized settings of conducted simulation scenarios in this paper.

Table 1. Simulation parameters

\begin{tabular}{|l|l|}
\hline Parameters & Value \\
\hline Simulation time & $700 \mathrm{~s}$ \\
\hline Simulation area & $2300 \times 1300 \mathrm{~m}$ \\
\hline Mobility model & Linear and Rectangle Models \\
\hline Number of MN & 50 \\
\hline MN Speed & Maximum $60 \mathrm{~km} / \mathrm{h}$ \\
\hline Transmitted Power WLAN & $14 \mathrm{dbm}$ \\
\hline Transmission range of each AP & $300 \mathrm{~meter}$ \\
\hline Maximum packet generation rate (real-time applications) & 1350 packet $/ \mathrm{second}$ \\
\hline Maximum packet size & 1000 byte \\
\hline Channel bandwidth WLAN & $11 \mathrm{Mbps}$ \\
\hline MAC protocol of WLAN & IEEE $802.11 \mathrm{~b} \mathrm{PCF}$ \\
\hline
\end{tabular}


Figure 3 depicts the average handover latency obtained each time the handover process occurred between MN and APs while FTP applications were run between MN and CN. In another words, the handover latency that occurred during a number of handovers performed by the $\mathrm{MN}$ during roaming process has been calculated as an average out of 10 simulation runs with FTP application. Figure 3, shows the average handover latency obtained by the AMH scheme during 5 handovers during simulation time with FTP application. The obtained latency with AMH scheme was $(0.19,0.17,0.21,0.26$, and $0.17 \mathrm{Sec})$ during simulation scenario time. The obtained average handover latency during the execution the FTP application with IF-HMIPv6 and SFMMA is $(0.79,0.8,0.95,0.99,1.2$, and $1.82 \mathrm{Sec})$ and $(0.99,1.1,1.65,1.85,1.92$, and 2.1 Sec) respectively based on 6 handovers. From the results in Figure 3 it can be seen that the handover latency with FTP application increased compared with the HTTP application. This is because the packet rate with FTP application increased compared to the HTTP which contributed to increasing the average handover latency. Furthermore, it can observed that the proposed AMH scheme could save the average obtained handover latency with FTP application a maximum value of $0.26 \mathrm{sec}$ compared with IF-HMIPv6 and SFMMA 1.82 and $2.1 \mathrm{sec}$ respectively.

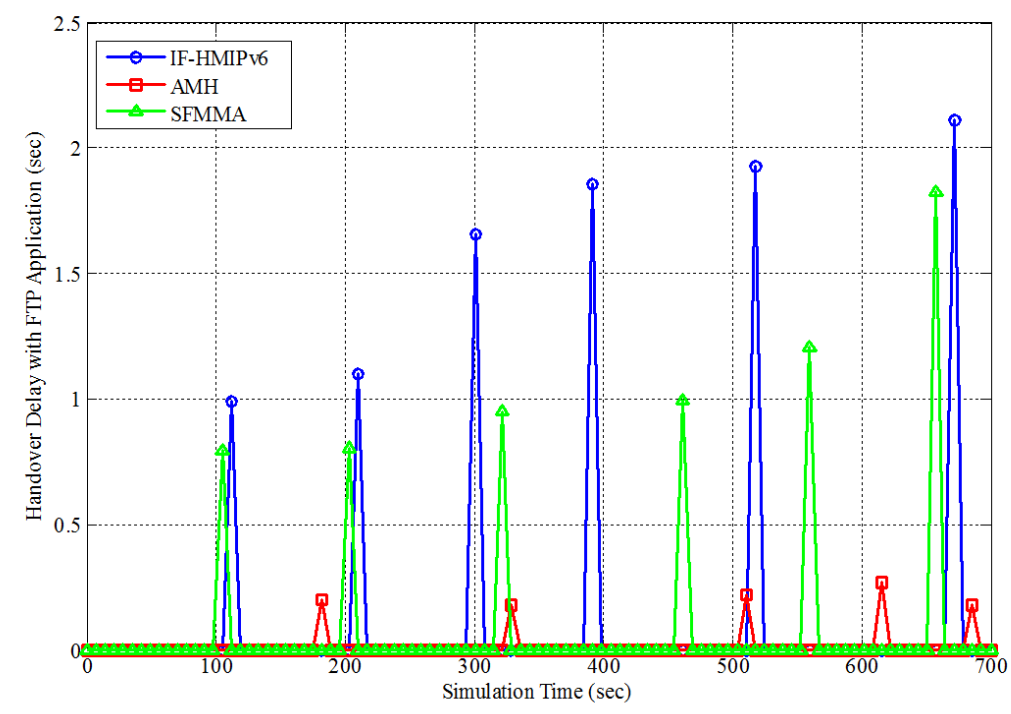

Figure 3. Average Handover Delay with FTP

Looking at Figure 4, the average handover latency obtained when the MN runs on the voice applications with CNs during its roaming process is illustrated. As can be seen, the collected average handover latency is depicted with $(5,6$, and 7) handover processes performed using AMH scheme, IF-HMIPv6, and SFMMA methods respectively with voice application. It is obvious that the handover latency with voice application increased sharply after $100 \mathrm{sec}$ with both IF-HMIPv6 and SFMMA during the handover process. However, the AMH scheme could successfully keep the handover latency at an average value that satisfies the voice application requirements. More precisely, by looking at Figure 4 it can be seen that the first handover occurred after 100 seconds with both IF-HMIPv6 and SFMMA methods with delays of 0.99 and $1.09 \mathrm{sec}$ respectively, whereas the obtained delay with the AMH scheme was $0.85 \mathrm{sec}$ after 217 seconds. The handover delay kept increasing with both IF-HMIPv6 and SFMMA methods during simulation time rising to $(1.3,1.84,2.2,2.8,2.82)$ and $(1.50,2.18,2.48,2.85,2.92,3.10)$ sec respectively. 


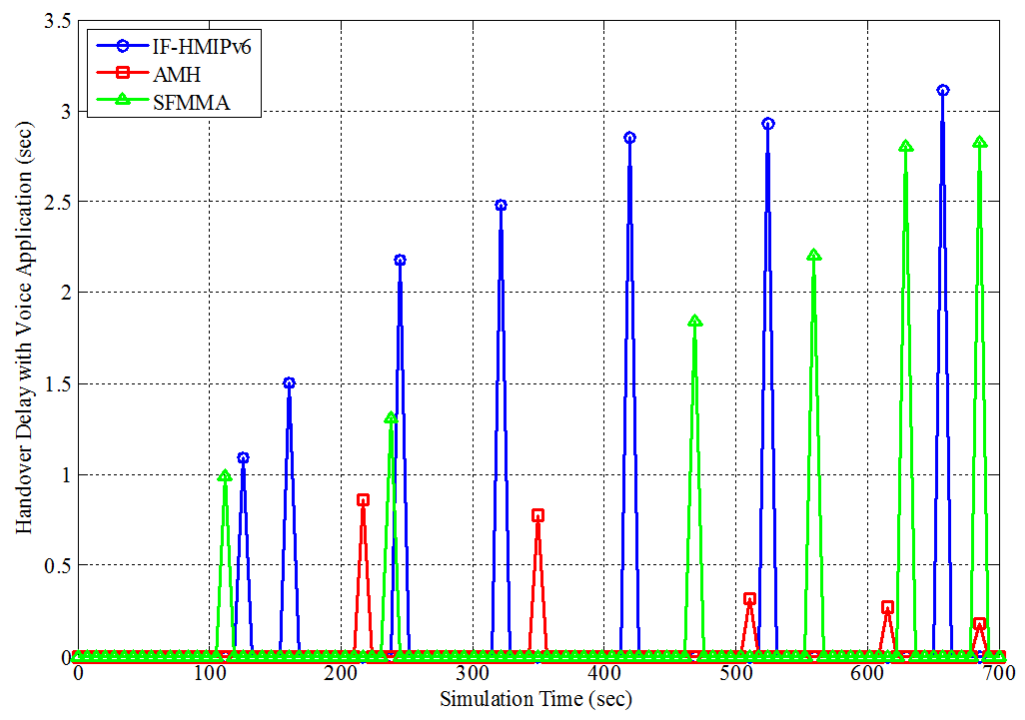

Figure 4. Average Handover Delay with voice

On the other hand, the handover delay using the proposed AMH scheme continuously deceased to $(0.77,0.31,0.26,0.17) \mathrm{sec}$ as shown in Figure 4. It is worth mentioning that the proposed AMH scheme essentially contributes to decreasing the handover delay with voice application compared with IF-HMIPv6 and SFMMA methods. Hence, the QoS voice application requirements performed in the session by $\mathrm{MN}$ are satisfied during the handover process with the AMH scheme with low time delay. Accordingly, the proposed AMH scheme could optimally achieve its objectives, by maintaining a VoIP session with low handover delay compared to the state of art.

\section{CONCLUSION}

In this paper, we proposed a developed network layer handover based wireless networks that perform the handover prior to its normal time, (after the handover in link layer has been processed). The mobility handovers were carried out by the next AP after the MN triggers the developed AMH message that was embedded with association_req frame during the association process with NAP. Thus, the handover in the network layer level was performed by NAP on behalf of the MN while it was still communicating with the current AP. Moreover, a unique IPv6 address was developed in this paper in order to keep the IPv6 address of the MNs during the simulated scenarios unchanged. The simulation results show that the developed network layer process performed the best in terms of decreasing the associated handover delay with FTP and Voice applications compare to the state of art.

\section{REFERENCES}

P. Brenner. A technical tutorial on the IEEE 802.11 protocol. BreezeCom Wireless Communications, 1992.

David Johnson, Charles Perkins, Jari Arkko, et al. Mobility support in ipv6, June 2004.

C. Perkins. Ip mobility support for ipv4. 2002.

David Johnson, Charles. Perkins, and Jari Arkko. Mobility support in ipv6, obsoletes rfc 3775, https://tools.ietf.org/html/rfc6275, July 2011. 
H. Soliman, L. Bellier, and K.E. Malki. Hierarchical mobile ipv6 mobility management (hmipv6), ietf standard obsoletes: 4140, http://xml2rfc.tools.ietf.org/html/rfc5380. October 2008.

H.J. Jang, Y.H. Han, J. Jee, S.D. Park, and J. Cha. Mobile ipv6 fast handovers over ieee 802.16 e networks.2008.

K.W. Lee, W.K. Seo, Y.Z. Cho, J.W. Kim, J.S. Park, and B.S. Moon. Inter-domain handover scheme using an intermediate mobile access gateway for seamless service in vehicular networks. International Journal of Communication Systems, 23(9-10):1127-1144, 2010.

Cheng-Wei Lee, Meng Chang Chen, and Yeali S Sun. Protocol and architecture supports for network mobility with qos-handover for high-velocity vehicles. Wireless Networks, pages 1-20, 2013.

P. Macha'n and J. Wo'zniak. Simultaneous handover scheme for ieee 802.11 wlans with ieee 802.21 triggers. Telecommunication Systems, 43(1):83-93, 2010.

Shayma Senan, Aisha Hassan A Hashim, A Saeed Rashid, and Jamal I Daoud. Evaluation of nested network mobility approaches. Applied Sci, 11:2244-2249, 2011.

Ilkwon Cho, Koji Okamura, Tae Wan Kim, and Choong Seon Hong. Performance analysis of ip mobility with multiple care-of addresses in heterogeneous wireless networks. Wireless Networks, pages 1-12, 2013.

Claude Castelluccia. Hmipv6: A hierarchical mobile ipv6 proposal. ACM SIGMOBILE Mobile Computing and Communications Review, 4(1):48-59, 2000.

MH Masud, F Anwar, SMS Bari, and OM Mohamed. Enhancement of handoff latency reduction mechanism of mobile internet protocol version 6 (mipv6). In Computer and Communication Engineering (ICCCE), 2012 International Conference on, pages 700705, Kuala Lumpur, 3-5 July 2012. IEEE.

J. Kempf. Problem statement for network-based localized mobility management (netlmm). 2007.

R. Koodli et al. Mobile IPv6 fast handovers, 2009.

V. Vassiliou and Z. Zinonos. An Analysis of the Handover Latency Components in Mobile IPv6. Journal of Internet Engineering, 3(1), 2010.

Sri Gundavelli, Kent Leung, Vijay Devarapalli, Kuntal Chowdhury, and Basavaraj Patil. Proxy mobile ipv6. Technical report, RFC 5213, August 2008.

Gerardo Giaretta. Interactions between proxy mobile ipv6 (pmipv6) and mobile ipv6 (mipv6. Technical report, RFC 6612, May 2012.

W. Chen-wen and W. Ping. Improved fast handover scheme for hierarchical mobile ipv6. In Computer Science \& Education, 2009. ICCSE'09. 4th International Conference on, pages 294-297. IEEE, 2009.

Rodolfo Ipolito Meneguette, Luiz Fernando Bittencourt, and Edmundo Roberto Mauro Madeira. A seamless flow mobility management architecture for vehicular communication networks. Journal of Communications and Networks, 15(2):207-216, 2013.

IEEE Computer Society. Wireless lan medium access control (mac) and physical layer specifications, 2007.

http://standards.ieee.org/getieee802/802.11.html. 